\title{
Erzeugung und Untersuchung biochemischer Mangelmutanten von Sacharomyces cerevisiae
}

\author{
Von F. Lingens und O. Oltmanns \\ Aus der Biochemischen Abteilung des Chemischen Institutes der Universität Tübingen \\ (Z. Naturforschg. 19 b, 1058-1065 [1964] ; eingegangen am 14. August 1964)
}

Die Wirkung von salpetriger Säure, UV-Bestrahlung, Methansulfonsäureäthylester (MSÄ) und Säure (verdünnte Schwefelsäure) auf Saccharomyces cerevisiae wurde untersucht.

Nach Behandlung mit salpetriger Säure traten etwa $1 \%$ auxotrophe Mutanten unter den Überlebenden auf, nach Behandlung mit UV-Licht 1-4 Prozent. MSÄ wirkte in saurem Medium stärker abtötend als in neutralem Medium. Die Mutationsraten lagen jedoch nach Einwirkung von MSÄ in neutraler Lösung bei gleichzeitig hohen Überlebendraten mit $4-5 \%$ am höchsten. Nach Behandlung mit Säure wurden keine Mutanten gefunden.

Der Nährstoffbedarf der Mutanten wurde ermittelt. Wachstumstests mit 3 Nicotinsäure-Mutanten lassen den Schluß zu, daß S. cerevisiae Nicotinsäure aus Tryptophan nach dem "Neurospora-Weg“" bildet. Auch Chinolinsäure ermöglicht diesen drei Mutanten das Wachstum.

Mit einer verbesserten Kreuzfütterungs-Methode wurde in einigen Fällen die Reihenfolge der genetischen Blockierung ermittelt.

Biochemische Mangelmutanten lassen sich mit Vorteil für die Aufklärung von Biosynthesewegen, für das Studium von Regulationsmechanismen und für genetische Untersuchungen verwenden. Sie können durch Strahlen und durch chemische Agentien induziert werden. Um eine möglichst hohe Ausbeute an Mutanten zu bekommen, verwendet man Agentien, die stark mutagen wirken, und eventuell Verfahren zur Mutantenanreicherung. Wir haben auf beiden Wegen versucht, Mutanten von Saccharomyces zu erhalten. In dieser Arbeit soll über die Einwirkung verschiedener Mutagene auf S. cerevisiae berichtet werden, in einer folgenden über Versuche zur Anreicherung von Hefemutanten.

Es gibt eine Fülle von Arbeiten über mutagene Agentien. Man kann trotzdem nicht ohne weiteres die „wirksamsten“ Mutagene aus den Angaben in der Literatur ermitteln, da die Mutationsraten vom Objekt und von der verwendeten Methodik abhängig sind. Wir haben drei bewährte Mutagene an unserem Hefestamm ausprobiert: Salpetrige Säure, ultraviolettes Licht (UV) und Methansulfonsäure-

${ }^{1}$ K. W. Mundry u. A. Gierer, Z. Vererbungslehre 89, 614 [1958].

2 R. K. Mortimer, R. S. Lerner u. J. K. Barr, U. S. Atomic Energy Commission, UCRL-3746 (1957).

3 A. Loveless u. S. Howarth, Nature [London] 184, 1780 [1959].

${ }^{4}$ H. Böнme, Biol. Zbl. 81, 267 [1962].

5 D. R. Krieg, Genetics 48,561 [1963].

${ }^{6}$ R. W. Kaplan, H. Beckmann u. W. Rüger, Nature [London] 199, 932 [1963]. äthylester - Äthylmethansulfonat - (MSÄ). Salpetrige Säure ist durch die Arbeit von Mundry und Gierer ${ }^{1}$ als mutagenes Agens eingeführt worden. UV-Strahlung ist bei Saccharomyces ein wirksames Mutagen, wie die Arbeit von Mortimer und Mitarbb. ${ }^{2}$ zeigte, die Mutationsraten zwischen 1,7 und $3,8 \%$ erreichten. MSÄ hat sich bei sehr vielen Organismen als hochwirksames Mutagen erwiesen. Es wurde im neutralen bis schwach sauren Bereich ${ }^{3-7}$, in ungepufferter, saurer wäßriger Lösung ${ }^{8-11}$ und als Aerosol ${ }^{12}$ angewandt. Wir haben die MSÄ-Wirkung bei $p_{\mathrm{H}}$-Werten von ca. 2, 5 und 7 verglichen. Außerdem untersuchten wir die abtötende und mutagene Wirkung von Säure (verd. Schwefelsäure), um den Anteil der Säure-Wirkung in den MSÄ. Versuchen abschätzen zu können.

Bisher sind relativ wenig Untersuchungen an biochemischen Mangelmutanten von Hefen beschrieben worden ${ }^{13,14}$. Bei der Aufklärung von Biosyntheseketten wurden einerseits Neurospora crassa und andererseits Enterobacteriaceen, vor allem Escherichia coli, herangezogen. Von Mortimer et al. ${ }^{2}$ wur-

7 G. Ivánovics, I. Varga u. E. Marjai, Naturwissenschaften 50, 674 [1963].

8 O. G. Fанмy u. M. J. FAHмy, Genetics 46, 1111 [1961].

9 J. L. Minocha u. T. J. Arnason, Nature [London] 196, 499 [1962].

10 G. Röbbelen, Naturwissenschaften 49, 65 [1962].

11 H. Gaul, Naturwissenschaften 49, 431 [1962].

12 E. A. Löвbеске u. R. C. von Borstel, Genetics 47, 853 [1962]. 
den Hefe-Mangelmutanten mit UV-Licht erzeugt. Über einige Untersuchungen daran berichteten wir früher ${ }^{15}$.

\section{Methodik}

Für alle Versuche verwendeten wir den haploiden Saccharomyces cerevisiae-Stamm S 288 C, den wir Herrn Dr. R. K. Mortimer, Berkeley, Californien, USA, verdanken.

Die Zuchttemperatur betrug $30{ }^{\circ} \mathrm{C}$. Folgende Medien wurden gebraucht:

Komplettmedium

\begin{tabular}{|c|c|c|c|}
\hline Pepton (Difco) & $10 \mathrm{~g}$ & Salzlösung & \\
\hline Hefeextrakt (Difco & o) $10 \mathrm{~g}$ & $\mathrm{NaCl}$ & $5,0 \mathrm{~g}$ \\
\hline Glucose oder & & $\mathrm{MgSO}_{4}+7 \mathrm{H}_{2} \mathrm{O}$ & $0,12 \mathrm{~g}$ \\
\hline Saccharose & $20 \mathrm{~g}$ & Aqua deion & $1000 \mathrm{ml}$ \\
\hline $\begin{array}{l}\text { Aqua deion } \\
\text { zur Verfestigung: }\end{array}$ & $1000 \mathrm{ml}$ & & \\
\hline Agar & $21 \mathrm{~g}$ & & \\
\hline Minimalmedium & [g] & Spurenlösung & {$[\mathrm{mg}]$} \\
\hline $\mathrm{KH}_{2} \mathrm{PO}_{4}$ & 0,875 & $\mathrm{H}_{3} \mathrm{BO}_{3}$ & 50 \\
\hline $\mathrm{K}_{2} \mathrm{HPO}_{4}$ & 0,125 & $\mathrm{CuSO}_{4}+5 \mathrm{H}_{2} \mathrm{O}$ & 4 \\
\hline $\mathrm{MgSO}_{4}+7 \mathrm{H}_{2} \mathrm{O}$ & 0,5 & $\mathrm{KJ}$ & 10 \\
\hline $\mathrm{NaCl}$ & 0,1 & $\mathrm{FeCl}_{3}+6 \mathrm{H}_{2} \mathrm{O}$ & 20 \\
\hline $\mathrm{CaCl}_{2}+6 \mathrm{H}_{2} \mathrm{O}$ & 0,1 & $\mathrm{MnSO}_{4}+4 \mathrm{H}_{2} \mathrm{O}$ & 40 \\
\hline$\left(\mathrm{NH}_{4}\right)_{2} \mathrm{SO}_{4}$ & 1,0 & $\mathrm{ZnSO}_{4}+7 \mathrm{H}_{2} \mathrm{O}$ & 40 \\
\hline Glucose & 10,0 & Ammonium- & \\
\hline $0 \mathrm{ml}$ von nebenst & tehender & molybdat & 20 \\
\hline purenlösung & & Biotin & 10 \\
\hline Aqua deion & $1000 \mathrm{ml}$ & Aqua dest. & $1000 \mathrm{ml}$ \\
\hline
\end{tabular}
zur Verfestigung:

Agar

$21 \mathrm{~g}$

Die Medien wurden 20 min bei 1 at autoklaviert.

$$
\text { Mutationsauslösung }
$$

Der Hefestamm S $288 \mathrm{C}$ wird 2 Tage auf Komplettagar angezogen. Zwei große Platinösen voll Hefezellen werden in $5 \mathrm{ml}$ Salzlösung suspendiert, 2-mal gewaschen und in $5 \mathrm{ml}$ Salzlösung in einer Konzentration von ca. $1,5 \cdot 10^{8}$ Zellen $/ \mathrm{ml}$ resuspendiert.

a) UV-Behandlung: Die Zellsuspension wird nach Bedarf verdünnt und auf Komplettagar plattiert. Die Platten werden anschließend mit einer UV-Niederdrucklampe (Osram HNS 12) bestrahlt und zur Vermeidung von Photoreversionen im Dunkeln inkubiert.

b) Behandlung mit salpetriger Säure: $3 \mathrm{ml}$ Zellsuspension werden mit vorgewärmten $3 \mathrm{ml}$ Acetatpuffer vom $p_{\mathrm{H}} 4,5$ und $3 \mathrm{ml} 0,1-m$. $\mathrm{NaNO}_{2}$ versetzt und in einem 50-ml-Kolben im Wasserbad unter Schütteln bei $30^{\circ}$ inkubiert.

13 Siehe G. Harris, in: The Chemistry and Biology of Yeasts, ed. A. H. Соок, Academic Press Inc., New York 1958.

14 Siehe P. Mandel u. R. Bieth, in: Die Hefen, Bd. I, Herausg. F. Reiff, R. Kautzmann, H. Lüers u. M. Lindemann, Verlag Hans Carl, Nürnberg 1960. c) Behandlung mit Methansulfonsäureäthylester: Die Zellen von $3 \mathrm{ml}$ Zellsuspension werden in vorgewärmten $9 \mathrm{ml}$ 0,2-m. MSÄ (Fa. Th. Schuchardt, München) suspendiert und in einem 50-ml-Kolben im Wasserbad unter Schütteln bei $30^{\circ}$ inkubiert.

MSÄ wurde gelöst: 1. in Aqua dest., worin sich nach der einstündigen Vorwärmzeit der $p_{\mathrm{H}-\text { Wert auf }}$ $2,2-1,8$ einstellte, 2. in Acetatpuffer von $p_{\mathrm{H}} 5$ (der $p_{\mathrm{H}}$-Wert sank während des Versuches auf 4,8$), 3$. in Phosphatpuffer von $p_{\mathrm{H}} 7$ (der $p_{\mathrm{H}}$-Wert sank während des Versuches bis auf 6,6).

d) Zum Vergleich werden die Hefezellen mit Säure $\left(n / 10-\mathrm{H}_{2} \mathrm{SO}_{4}\right)$ wie oben im Wasserbad inkubiert.

Den Inkubationsgemischen $\mathrm{b}, \mathrm{c}$ und $\mathrm{d}$ werden $\mathrm{zu}$ verschiedenen Zeiten Proben entnommen. Diese werden jeweils so stark mit Salzlösung verdünnt, daß nach dem Plattieren auf Komplettagar möglichst ca. 20 bis 100 Kolonien je Platte wachsen.

$$
\text { Isolierung von Mutanten }
$$

Die mit den behandelten Zellen bedeckten Komplettagarplatten werden $3-4$ Tage inkubiert. Von den Hefekolonien wird dann eine Probe mit einer Platinöse abgenommen und zuerst auf Minimalagar, danach auf Komplettagar ausgestrichen. Die mutmaßlichen Mutanten, die nach 3 Tagen auf Komplettagar aber nicht auf Minimalagar gewachsen sind, werden in zwei weiteren solcher Strichtests auf Konstanz ihres Nährstoffbedarfs geprüft. Sie werden anschließend auf Schrägröhrchen übergeführt und im Eisschrank aufbewahrt. Eine Übertragung auf frische Schrägröhrchen erfolgt zweckmäßig alle 6-10 Monate.

\section{Identifizierung der Mutanten}

a) Blättchentest: Die Mutanten werden über Nacht in $5 \mathrm{ml}$ Komplettmedium angezüchtet, 2-mal mit Salzlösung gewaschen, auf ca. $10^{7}$ Zellen $/ \mathrm{ml}$ verdünnt und in $5 \mathrm{ml}$ Salzlösung $6 \mathrm{Stdn}$. inkubiert. Die Suspension wird in sterile Petrischalen gegossen und mit $15 \mathrm{ml}$ handwarmem Minimalagar vermischt. Nach einer Stde. werden 7 Filtrierpapierblättchen aufgelegt, die mit verschiedenen Gruppen von Nährstoffen (s. Tab. 2) getränkt und bei $80^{\circ}$ getrocknet waren. Im Durchschnitt enthalten die Blättchen an (1-) Aminosäuren und $\mathrm{Nu}$ cleobasen jeweils $50 \gamma$, an Threonin $500 \gamma$ und an Vitaminen $5 \gamma$. Die Nährstoffzusammenstellung in den einzelnen Gruppenblättchen ist gegenüber l. c. ${ }^{16}$ verbessert und mehr den biosynthetischen Zusammenhängen der einzelnen Stoffe angeglichen worden.

Nach einigen Tagen bildet sich in der Regel um eines der Gruppenblättchen ein Wachstumshof. Die Mutanten werden einem zweiten Blättchentest unterworfen. Dieses Mal wird das wachstumsfördernde Gruppenblättchen aufgelegt und außerdem Blättchen, die nur

15 F. Lingens u. W. Lück, Hoppe-Seyler's Z. physiol. Chem. 333,190 [1963]. 
mit je einem Stoff dieses Gruppenblättchens versehen sind. Das wachstumsfördernde Einzelblättchen zeigt den Bedarf der Mutante an. Es kommt vor, daß Wachstum nicht in einem Hof um ein Blättchen herum auftritt, sondern zwischen zwei benachbarten Blättchen (z. B. zwischen Tyrosin und Phenylalanin) oder nur in einem Hof um das Gruppenblättchen (z. B. bei polyauxotrophen Mutanten der Aromaten-Biosynthesekette).

b) Wachstumstest in flüssigem Medium: Die Mutanten werden über Nacht in $5 \mathrm{ml}$ Komplettmedium angezogen, 2-mal gewaschen und in $5 \mathrm{ml}$ Salzlösung suspendiert. Je ein Tropfen Hefesuspension kommt in ein Röhrchen mit $5 \mathrm{ml}$ Minimalmedium, dem die auf Wachstumswirksamkeit zu prüfenden Stoffe zugesetzt sind, z. B. Tryptophan, Indol, Anthranilsäure bei Tryptophan-Mangelmutanten. Aminosäuren und Aminosäure-Vorstufen werden in einer Konzentration von $20 \gamma / \mathrm{ml}$ angewendet, Vitamine in einer Konzentration von $2 \gamma / \mathrm{ml}$. Labile Substanzen, z. B. Vitamin-Vorstufen, werden nicht autoklaviert, sondern in einer Lösung von sterilem Aqua dest. zugegeben. Bei Vitamin-bedürftigen Mutanten ist es nötig, die Hefezellen durch eine Passage in Minimalmedium an Vitamin $\mathrm{zu}$ verarmen und erst dann im Flüssigtest zu prüfen.

c) Kreuzfütterungstest: Mehrere Mutanten mit gleichem Nährstoffbedarf werden, in Abwandlung der Methode von Gibson et al. ${ }^{17}$, über Nacht in je $5 \mathrm{ml} \mathrm{Kom-}$ plettmedium angezogen, 2-mal gewaschen und in $4 \mathrm{ml}$ Salzlösung suspendiert. Jeweils $3 \mathrm{ml}$ dieser Suspensionen werden in sterilen Petrischalen mit $15 \mathrm{ml}$ handwarmem Minimalagar vermischt. Die Platten werden einen Tag lang bebrütet. Danach werden die restlichen 1-ml-Suspensionen 1 : 5 verdünnt und Proben von jeder

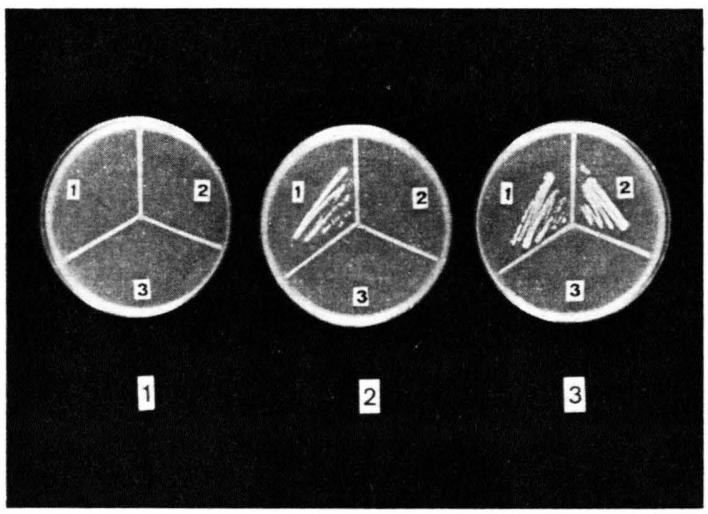

Abb. 1. Kreuzfütterungstest mit drei an verschiedenen Stellen des Tryptophan-Biosyntheseweges blockierten Mangelmutanten. Diese Mutanten benötigen zum Wachstum: Anthranilsäure, Indol oder Tryptophan 1; Indol oder Tryptophan 2 bzw. Tryptophan 3. Durch das Akkumulat der im Minimalagar suspendierten Mutante 1 (linke Platte) wird keine der drei in Suspension aufgetragenen Mutanten zum Wachstum gebracht. Die Mutante 2 (mittlere Platte) füttert mit ihrem akkumulierten Tryptophan-Vorprodukt die Mutante 1, die

Mutante 3 (rechte Platte) füttert die Mutanten 1 und 2.
Mutante auf jeder Minimalagarplatte aufgetragen. Mit 10 Minimalagarplatten, die je eine Mutante und deren Akkumulat enthalten, lassen sich auf diese Weise 10 Mutanten gegeneinander austesten. Wenn eine in Suspension auf dem Agar aufgetragene Mutante wächst, bedeutet das, daß sie ihren genetischen Block vor dem der akkumulierenden Mutante hat (Abb. 1).

Durch eine leichte Variation dieser Versuchsanordnung lassen sich verschiedene Organismenarten gegeneinander testen: Man läßt zum Beispiel try ${ }^{-}$-Mutanten von $E$. coli in $5 \mathrm{ml}$ flüssigem Bakterienminimalmedium 1 Tag akkumulieren und vermischt diese Suspension dann mit $15 \mathrm{ml}$ Hefeminimalagar, auf den nach dem Erkalten try ${ }^{-}$-Mutanten von $S$. cerevisiae in Suspension aufgetragen werden.

\section{Ergebnisse}

\section{Einwirkung mutagener Agentien}

Beispiele von Abtötungskurven sind in Abb. $2 \mathrm{a}-\mathrm{f}$ aufgeführt. Die Neigung der Geraden variiert bei jedem Agens etwas von Versuch zu Versuch. Auch der Knick in den Kurven ist je nach Versuch mehr oder weniger stark ausgeprägt. Die abtötende Wirkung des Methansulfonsäureäthylesters ist deutlich vom $p_{\mathrm{H}}$-Wert der Lösung abhängig, sie ist am geringsten im neutralen $p_{\mathrm{H}}$-Bereich. Daß im sauren Bereich nicht nur eine Inaktivierung durch die höhere Wasserstoffionenkonzentration vorliegt, zeigt ein Vergleich mit der Abtötung der Hefen durch $n / 10-\mathrm{H}_{2} \mathrm{SO}_{4}$ (Abb. $\left.2 \mathrm{f}\right)$.
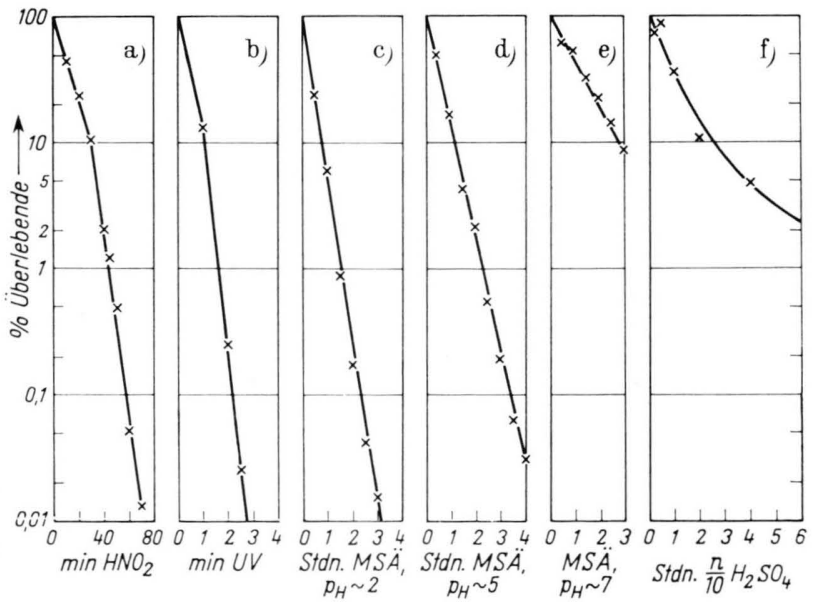

Abb. 2. Abtötung von S. cerevisiae S $288 \mathrm{C}$ durch (a) 0,033-m. salpetrige Säure, $p_{\mathrm{H}}$ 4,5, (b) UV-Licht, (c) 0,2-m. MSÄ, $p_{\mathrm{H}} 2,2-1,8$, (d) $0,2-m$. MSÄ, $p_{\mathrm{H}} 5,0-4,8$, (e) $0,2-m$. MSÄ, $p_{\mathrm{H}} 7,0-6,6$, (f) $n / 10-\mathrm{H}_{2} \mathrm{SO}_{4}$. Ausgangs-Zellkonzentration ca. $5 \cdot 10^{7}$ Zellen $/ \mathrm{ml}$.

17 F. Gibson u. M. J. Jones, Austral. J. Sci. 17, 33 [1954].

${ }^{16}$ F. Lingens, Z. Naturforschg. 19 b,151 [1964]. 
Die Mutationsraten sind aus Tab. 1 zu entnehmen. Nach Nitritbehandlung erhielten wir etwa $1 \%$ Mutanten unter den Überlebenden, nach UV-Behandlung zwischen 1 und $4 \%$ und nach MSÄ-Behandlung zwischen 1 und 5 Prozent. Es fällt auf, daß in den UV-Versuchen die Mutationsrate mit der Abtötungsrate zunimmt, daß jedoch nach MSÄ-Behandlung hohe Mutationsraten auch bei niedrigen Abtötungswerten erhalten werden. Konstant hohe Mutationsraten, nämlich 3,0 und $4,75 \%$ wurden nach MSÄ-Behandlung im neutralen $p_{\mathrm{H}}$-Bereich erzielt. Auch in weiteren Versuchen erwies sich dieser $p_{\mathrm{H}}$-Bereich als günstig für die Erzielung hoher $\mathrm{Mu}$ tationsraten: Nach 2-stdg. Einwirkung von MSÄ (Überlebendrate ca. 20\%) waren in einem Falle unter 150 geprüften Kolonien 6 Mutanten, in einem anderen Falle unter 200 Kolonien 15, in einem weiteren unter 200 Kolonien 9 Mutanten.

Die Verteilung der Mutanten hinsichtlich ihres Nährstoffbedarfes geben wir in Tab. 2 an. In dieser
Tabelle sind neben den Mutanten aus Tab. 1 weitere Mutanten enthalten, die mit abweichender Methodik erhalten wurden. In einigen Versuchen wurde z. B. bei der Isolierung der Mutanten die L e d e r b e r g Stempeltechnik angewendet, in anderen Versuchen wurde der Wildstamm für die Mutationsversuche in flüssigem Komplettmedium statt auf Komplettagar angezogen. In diesen Versuchen erzielten wir stets niedrigere Mutationsraten als mit der angegebenen Standard-Methode.

Einige der Mutanten, besonders thiaminbedürftige, sind durch den angezeigten Stoff nur schlecht zum Wachstum zu bringen. Andere können wahlweise mit mehreren Stoffen wachsen. Das ist erklärlich bei Wachstum mit biochemisch verwandten Stoffen wie Glutaminsäure und Glutamin, Methionin und Cystin, Adenin und Guanin, aber bisher nicht erklärbar bei Wachstum mit Uracil oder Inosit; Methionin oder Inosit; Thiamin, Inosit oder Pantothensäure; Inosit, Leucin oder Valin; Pyridoxin

\begin{tabular}{|c|c|c|c|c|c|c|}
\hline \multirow[b]{2}{*}{ Mutagenes Agens } & \multirow{2}{*}{$\begin{array}{l}\text { Zahl der } \\
\text { Versuche }\end{array}$} & \multirow{2}{*}{$\begin{array}{l}\text { Behand- } \\
\text { lungsdauer }\end{array}$} & \multirow{2}{*}{$\begin{array}{c}\text { Überlebende } \\
{[\%]}\end{array}$} & \multirow{2}{*}{$\begin{array}{c}\text { Kolonien } \\
\text { getestet }\end{array}$} & \multicolumn{2}{|c|}{ Mutanten } \\
\hline & & & & & gefunden & $\begin{array}{c}\% \mathrm{~d} . \\
\text { Úberl. }\end{array}$ \\
\hline \multirow{6}{*}{$\begin{array}{l}0,033-\mathrm{m} . \\
\text { salpetrige Säure }\end{array}$} & 1 & $25 \mathrm{~min}$ & 7,8 & 427 & 3 & 0,7 \\
\hline & 1 & & 1,44 & 300 & 1 & 0,33 \\
\hline & 2 & 50 & 0,71 & 900 & 13 & 1,45 \\
\hline & 2 & 55 & 0,36 & 640 & 6 & 0,94 \\
\hline & 1 & 60 & 0,20 & 280 & 1 & 0,36 \\
\hline & & & & 2547 & 24 & $\overline{0,94}$ \\
\hline \multirow{6}{*}{$\begin{array}{l}\text { ultra-violettes } \\
\text { Licht }\end{array}$} & 3 & $1 \mathrm{~min}$ & 14,7 & 540 & 3 & 0,56 \\
\hline & 3 & & 0,58 & 620 & 9 & 1,45 \\
\hline & 3 & 2,5 & 0,056 & 568 & 21 & 3,7 \\
\hline & 2 & 3 & 0,0034 & 290 & 10 & 3,45 \\
\hline & 1 & 3,5 & 0,001 & 100 & 4 & 4,0 \\
\hline & & & & 2118 & $\overline{47}$ & $\overline{2,22}$ \\
\hline \multirow{6}{*}{$\begin{array}{l}0,2-\mathrm{m} \text {. Methan- } \\
\text { sulfonsäure- } \\
\text { äthylester } \\
p_{\mathrm{H}} 2,2-1,8\end{array}$} & 1 & $0,5 \mathrm{Stdn}$. & 39,2 & 300 & 5 & 1,67 \\
\hline & 1 & 0,75 & 23,3 & 500 & 22 & 4,4 \\
\hline & 1 & 2,5 & 1,22 & 412 & 6 & 1,45 \\
\hline & 1 & 3 & 0,29 & 849 & 10 & 1,18 \\
\hline & 1 & 3,5 & 0,061 & 317 & 6 & 1,9 \\
\hline & & & & $\overline{2378}$ & 49 & 2,06 \\
\hline \multirow{6}{*}{$\begin{array}{l}0,2-\mathrm{m} . \mathrm{MSÄ} \\
p_{\mathrm{H}} 5,0-4,8\end{array}$} & 1 & $0,5 \mathrm{Stdn}$. & 40,0 & 481 & 11 & 2,29 \\
\hline & 2 & 2,5 & 1,29 & 1043 & 12 & 1,15 \\
\hline & 2 & 3 & 0,81 & 1167 & 17 & 1,46 \\
\hline & 1 & 3,5 & 0,57 & 424 & 16 & 3,6 \\
\hline & 1 & 4 & 0,25 & 447 & 7 & 1,57 \\
\hline & & & & $\overline{3562}$ & $\overline{63}$ & 1,77 \\
\hline $0,2-\mathrm{m} . \mathrm{MSÄ}$ & 1 & $2 \mathrm{Stdn}$ & 49,5 & 379 & 18 & 4,75 \\
\hline$p_{\mathrm{H}} 7,0-6,6$ & 1 & 3 Stdn. & 14,7 & $\begin{array}{l}567 \\
946\end{array}$ & $\begin{array}{l}17 \\
35\end{array}$ & $\begin{array}{l}3,0 \\
3,70\end{array}$ \\
\hline$n / 10-\mathrm{H}_{2} \mathrm{SO}_{4}$ & 2 & $10 \mathrm{Stdn}$ & 1,3 & 890 & $\mathbf{0}$ & $\leqq 0,11$ \\
\hline $\begin{array}{l}\text { Kontrolle } \\
\text { (ohne Behandlung) }\end{array}$ & 15 & - & 100,0 & 2535 & 0 & $\leqq \mathbf{0 , 0 3 9}$ \\
\hline
\end{tabular}

Tab. 1. Häufigkeit der Mutanten unter den Überlebenden in Abhängigkeit vom mutagenen Agens und von der Überlebendrate. 


\begin{tabular}{|c|c|c|c|c|c|}
\hline \multirow[b]{2}{*}{ Gruppe } & \multirow[b]{2}{*}{ Nährstoff } & \multicolumn{4}{|c|}{ Anzahl der Mutanten nach Einwirkung von } \\
\hline & & $\begin{array}{l}\text { salpetriger } \\
\text { Säure }\end{array}$ & UV-Licht & $\begin{array}{l}\text { Methan- } \\
\text { sulfonsäure- } \\
\text { äthylester }\end{array}$ & Summe \\
\hline 1 & $\begin{array}{l}\text { Cystin } \\
\text { Methionin } \\
\text { Serin } \\
\text { Glycin }\end{array}$ & $\begin{array}{r}- \\
13 \\
1 \\
-\end{array}$ & $\begin{array}{l}\overline{-} \\
\overline{5} \\
-\end{array}$ & $\begin{array}{r}1 \\
28 \\
1 \\
1\end{array}$ & $\begin{array}{r}1 \\
46 \\
2 \\
1\end{array}$ \\
\hline 2 & $\begin{array}{l}\text { Leucin } \\
\text { Isoleucin } \\
\text { Valin } \\
\text { Threonin } \\
\text { Lysin }\end{array}$ & $\begin{array}{l}1 \\
- \\
- \\
- \\
3\end{array}$ & $\begin{array}{r}1 \\
1 \\
1 \\
-4\end{array}$ & $\begin{array}{r}8 \\
1 \\
- \\
2 \\
9\end{array}$ & $\begin{array}{r}10 \\
2 \\
1 \\
2 \\
16\end{array}$ \\
\hline 3 & $\begin{array}{l}\text { Tyrosin } \\
\text { Phenylalanin } \\
\text { Tryptophan } \\
p \text {-Aminobenzoesäure } \\
\text { Pyridoxin-HCl } \\
\text { Nicotinsäureamid }\end{array}$ & $\begin{array}{c}3 \\
- \\
2 \\
-7 \\
1\end{array}$ & $\begin{array}{l}\frac{2}{-} \\
2 \\
- \\
-\end{array}$ & $\begin{array}{c}2 \\
1 \\
6 \\
-4 \\
4 \\
2\end{array}$ & $\begin{array}{r}7 \\
1 \\
10 \\
-16 \\
3\end{array}$ \\
\hline 4 & $\begin{array}{l}\text { Glutamin } \\
\text { Glutaminsäure } \\
\text { Asparagin } \\
\text { Asparaginsäure } \\
\text { Alanin } \\
\text { Prolin }\end{array}$ & $\begin{array}{l}1 \\
(1) \\
1 \\
(1) \\
- \\
-\end{array}$ & $\begin{array}{l}- \\
- \\
1 \\
(1) \\
- \\
-\end{array}$ & $\begin{array}{l}- \\
- \\
- \\
- \\
-\end{array}$ & $\begin{array}{l}1 \\
(1) \\
2 \\
(2) \\
- \\
-\end{array}$ \\
\hline 5 & $\begin{array}{l}\text { Thiamin } \\
\text { Riboflavin } \\
\text { Ca-Pantothenat } \\
\text { Inosit } \\
\text { Folsäure } \\
\text { Cobalamin }\end{array}$ & $\begin{array}{r}27 \\
- \\
4 \\
15 \\
- \\
-\end{array}$ & $\begin{array}{c}9 \\
- \\
4 \\
9 \\
- \\
-\end{array}$ & $\begin{array}{r}20 \\
\frac{7}{7} \\
20 \\
- \\
-\end{array}$ & $\begin{array}{l}56 \\
\frac{56}{15} \\
44 \\
- \\
-\end{array}$ \\
\hline 6 & $\begin{array}{l}\text { Adenin } \\
\text { Guanin } \\
\text { Thymin } \\
\text { Uracil } \\
\text { Arginin } \\
\text { Histidin }\end{array}$ & $\begin{array}{c}9 \\
(1) \\
- \\
3 \\
6 \\
4\end{array}$ & $\begin{array}{l}3 \\
(1) \\
- \\
5 \\
5 \\
3\end{array}$ & $\begin{array}{l}13 \\
(1) \\
- \\
5 \\
4 \\
9\end{array}$ & $\begin{array}{l}25 \\
(3) \\
- \\
13 \\
15 \\
16\end{array}$ \\
\hline $1-6$ & & 5 & 2 & 13 & 20 \\
\hline $\begin{array}{l}\text { Poly- } \\
\text { auxo- } \\
\text { trophe } \\
\text { Mutanten }\end{array}$ & $\begin{array}{l}\text { Tyr }+ \text { Phe } \\
\text { Tyr+Phe }+ \text { Try }+ \text { pAbs } \\
\text { Meth + Threo } \\
\text { Meth + Lys } \\
\text { Threo+Lys } \\
\text { Ileu+Val } \\
\text { His + Arg } \\
\text { His + Ade }\end{array}$ & $\begin{array}{l}1 \\
3 \\
1 \\
- \\
- \\
- \\
-\end{array}$ & $\begin{array}{l}- \\
1 \\
- \\
- \\
- \\
- \\
-\end{array}$ & $\begin{array}{l}1 \\
7 \\
1 \\
1 \\
1 \\
1 \\
1 \\
1\end{array}$ & $\begin{array}{r}2 \\
11 \\
2 \\
1 \\
1 \\
1 \\
1 \\
1\end{array}$ \\
\hline \multicolumn{2}{|c|}{ Komplettmedium } & $\frac{2}{113}$ & $\frac{1}{64}$ & $\begin{array}{r}4 \\
175\end{array}$ & $\begin{array}{r}7 \\
352\end{array}$ \\
\hline
\end{tabular}

Tab. 2. Nährstoffbedarf der isolierten Mutanten. Die Nährstoffzusammensetzung in den Gruppen 1 bis 6 entspricht der in den Blättchen für den ersten Blättchentest (s. Methodik). Das Blättchen „1-6“ enthält alle verwendeten Substanzen. Zahlen in Klammern bezeichnen Mutanten, die wegen ihres Wachstums mit einer anderen Substanz in der Endsumme nicht noch einmal erscheinen.

oder Methionin; Nicotinsäure oder Tyrosin. Diese Mutanten sind in der Tab. 2 nur einmal aufgeführt.

\section{Untersuchungen an einigen Mutantengruppen}

Die Lysin-Mutanten und die Uracil-Mutanten ließen sich durch Kreuzfütterungstests in je zwei
Gruppen aufteilen, von denen die eine Gruppe die andere füttert, also später blockierte Mutanten umfaßt.

Bei Vitamin-Mutanten ergaben Kreuzfütterungstests bisher keine eindeutigen Resultate.

Die drei Nicotinsäure-Mutanten ließen sich durch 
Wachstumstests näher bestimmen (Tab. 3). Alle drei Mutanten wachsen mit Nicotinsäureamid und mit Chinolinsäure. Zwei Mutanten wachsen außerdem auch mit Tyrosin, wofür wir bisher keine Erklärung haben. Eine Mutante wächst mit Nicotinsäureamid, Chinolinsäure und 3-Hydroxyanthranilsäure, eine weitere mit diesen drei Stoffen und mit Kynureninsulfat. Aus diesen Wachstumstests läßt sich ableiten, daß die drei Mutanten an verschiedenen Stellen des Nicotinsäure-Syntheseweges blockiert sind, und zwar im Syntheseschritt zur Chinolinsäure (HK 84), zur 3-Hydroxyanthranilsäure (E 105) und zum Kynurenin (HK 14).

\begin{tabular}{|c|c|c|c|}
\hline $\begin{array}{c}\text { Minimalmedium } \\
+ \text { Zusatz von je } 2 \gamma / \mathrm{ml}\end{array}$ & HK 84 & $\begin{array}{l}\text { Mutante } \\
\text { E } 105\end{array}$ & HK 14 \\
\hline $\begin{array}{l}\text { Nicotinsäureamid } \\
\text { Chinolinsäure } \\
\text { 3-Hydroxyanthranil- } \\
\text { säure } \\
\text { Kynureninsulfat } \\
\text { Tyrosin } \\
0\end{array}$ & $\begin{array}{r}59 \\
44 \\
\\
7 \\
7 \\
35 \\
8\end{array}$ & $\begin{array}{r}65 \\
65 \\
65 \\
5 \\
5 \\
6\end{array}$ & $\begin{array}{c}46 \\
46 \\
\\
31 \\
45 \\
5 * \\
6\end{array}$ \\
\hline
\end{tabular}

Tab. 3. Wachstum von Nicotinsäure-Mutanten mit Nicotinsäure-Vorstufen und Tyrosin. Trübungsmessungen mit dem B. Lange-Kolorimeter nach 4 Tagen Wachstum. Die Zahlen bedeuten Skalenteile. * $20 \gamma$ Tyrosin $/ \mathrm{ml}$ ermöglichen dieser Mutante schwaches Wachstum.

Wegen der besonderen Bedeutung der Fütterung von Nicotinsäure-Mutanten durch Chinolinsäure (s. Diskussion) haben wir diese Substanz auch in geringeren Konzentrationen getestet als in der Methodik angegeben wurde (Tab. 4). Die Mutanten E 105 und HK 14 können Chinolinsäure in den gleichen niedrigen Konzentrationen verwerten wie Nicotinsäureamid. Bei der Mutante HK 84 fördert Chinolinsäure das Wachstum weniger gut, sie besitzt nur wenige Prozent der Wirksamkeit von Nicotinsäure bzw. Nicotinsäureamid (Tab. 5). Diese Wirksamkeit kann nur z. T. von Verunreinigungen der Chinolinsäure herrühren, denn Tests mit Lactobacillus arabinosus in Verbindung mit papierchromatographi-

\begin{tabular}{|l|r|r|r|r|r|r|r|}
\hline \multirow{2}{*}{ Mutante } & \multicolumn{3}{|c|}{$\begin{array}{c}\text { Nicotinsäureamid } \\
{[\gamma / \mathrm{ml}]}\end{array}$} & \multicolumn{3}{c|}{$\begin{array}{c}\text { Chinolinsäure } \\
{[\gamma / \mathrm{ml}]}\end{array}$} & 0 \\
& 0,5 & 0,05 & 0,01 & 0,5 & 0,05 & 0,01 & \\
& & & & & & \\
HK 84 & 46 & 15 & 4 & 4 & 5 & 4 & 5 \\
E 105 & 65 & 62 & 37 & 67 & 58 & 29 & 4 \\
HK 14 & 40 & 37 & 23 & 40 & 31 & 13 & 5 \\
\hline
\end{tabular}

Tab. 4. Vergleich der wachstumsfördernden Wirkung von Nicotinsäureamid und Chinolinsäure auf Nicotinsäure-Mutanten. Trübungsmessungen mit dem B. Lange-Kolorimeter nach 4 Tagen Wachstum. Die Zahlen bedeuten Skalenteile. scher Reinigung der Chinolinsäure ergaben für die eingesetzte Chinolinsäure nur einen Anteil von $0,04 \%$ Nicotinsäure und von $0,14 \%$ einer zweiten Lactobacillus arabinosus fütternden Substanz mit $\operatorname{dem} R_{f}$-Wert von Chinolinsäure.

\begin{tabular}{|c|c|c|c|c|c|c|c|}
\hline Mutante & 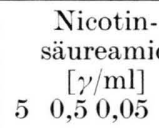 & id & $\begin{array}{l}\text { Nicotin- } \\
\text { säure } \\
{[\gamma / \mathrm{ml}]} \\
0,05\end{array}$ & $\begin{array}{ll}\text { Chin } & \\
5 & 0,2\end{array}$ & $\mathrm{ml}$ & $\begin{array}{l}\text { iure } \\
0,01\end{array}$ & 0 \\
\hline HK 84 & $\begin{array}{lll}55 & 54 & 22\end{array}$ & 6 & 22 & 487 & 6 & 6 & 6 \\
\hline
\end{tabular}

Tab. 5. Wachstum der Nicotinsäure-Mutante HK 84 mit verschiedenen Konzentrationen von Nicotinsäureamid, Nicotinsäure und Chinolinsäure. Trübungsmessungen mit dem B. Lange-Kolorimeter nach 5 Tagen Wachstum. Die Zahlen bedeuten Skalenteile.

Auch die 10 Tryptophan-Mutanten wurden näher analysiert. Drei dieser Mutanten wachsen mit Anthranilsäure, Indol und Tryptophan, 5 wachsen mit Indol und Tryptophan und 2 weitere wachsen nur mit Tryptophan. Kreuzfütterungstests ergaben Resultate, die diesem dreistufigen Schema entsprechen (s. a. Abb. 1) : Mutanten der ersten Gruppe (genetischer Block vor Anthranilsäure) wachsen mit den Akkumulaten der Mutanten der zweiten und dritten Gruppe, Mutanten der zweiten Gruppe (genetischer Block vor dem Indol) wachsen nur mit den Akkumulaten der Mutanten der dritten Gruppe. Eine der Indol-bedürftigen Mutanten füttert jedoch nicht die Anthranilsäure ${ }^{-}$-Mutanten. Es gibt Hinweise dafür, daß bei dieser Mutante mehrere Gene inaktiv geworden sind. Die Untersuchung der akkumulierten Zwischenprodukte der Mutanten ist im Gange.

Elf polyauxotrophe Aromaten-Mutanten mit 4-fachem Bedarf wurden isoliert (Tab. 2). Keine dieser Mutanten war mit Shikimisäure zum Wachstum zu bringen. Auch Kreuzfütterungstests verliefen negativ. Die Mutanten wachsen in der ersten Passage in Minimalmedium plus Tyrosin, Phenylalanin und Tryptophan. Nach der Übertragung in frisches Minimalmedium muß neben diesen drei Aminosäuren noch $p$-Aminobenzoesäure zugesetzt werden, damit weiteres Wachstum vonstatten gehen kann. Mutanten mit Bedarf an den drei aromatischen Aminosäuren, wie sie bei Bakterien beschrieben wurden ${ }^{18}$, haben wir nicht gefunden.

\section{Diskussion}

Haploide Zellen sollten theoretisch im halblogarithmischen Raster geradlinige Abtötungskurven er18 B. D. DAvis, J. Bacteriol. 64, 729 [1952]. 
geben, vorausgesetzt, daß die Schädigung eines DNS. Stranges zur Inaktivierung ausreicht. Der mehr oder weniger deutliche Knick in unseren Kurven (Abb. 2) könnte durch einen gewissen Anteil von mehrkernigen Zellen bzw. von Zellhaufen erklärt werden oder durch ein nur langsames Eindringen bzw. verzögertes Wirksamwerden des inaktivierenden Agens. Wir haben diese Verhältnisse nicht näher untersucht.

Die abtötende Wirkung von Methansulfonsäureäthylester ist um so größer, je saurer die Behandlungslösung ist. Die höchsten Mutationsraten (von $4-5 \%$ ) werden dagegen mit neutraler Behandlungslösung erreicht (Tab. 1).

Vergleichbare Ergebnisse erhielten Froese-Gertzen und Mitarb. in ihren Untersuchungen an Gerste ${ }^{19,} 20$. Die Keimlingshöhe von Gerste wird nach Behandlung der Samen mit ungepufferter MS ̈̈-Lösung stär-

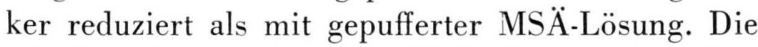
schädigende Wirkung der Hydrolyseprodukte Methansulfonsäure und Äthylalkohol kann durch Zusatz von Puffer vermindert werden. Nach vorläufigen Untersuchungen soll die (mutagene) Wirksamkeit durch Pufferung der Behandlungslösung erhöht werden können ${ }^{20}$.

Auf die hohen MSÄ-induzierten Mutationsraten bei gleichzeitig hohen Überlebendraten hat zum ersten Male Loveless ${ }^{3}$ hingewiesen, der Bakterien und Phagen mit MSÄ in Puffer behandelte. Eine gleichartige Erscheinung wurde bei anderen Agentien bisher nicht beobachtet. Systematische Untersuchungen zu dieser Frage liegen jedoch nicht vor.

Nächst MSÄ erbrachte UV-Bestrahlung die höchsten Mutationsraten $(1-4 \%)$. Sie liegen in unseren Versuchen nicht ganz so hoch wie bei Mortimer und Mitarbb. ${ }^{2}$, wenn man berücksichtigt, daß diese Vitamin-Mutanten nicht erfaßt haben, die in unseren Versuchen etwa $1 / 4$ aller Mutanten ausmachen.

Nach Nitritbehandlung erhielten wir nur ca. 1\% Mutanten unter den Überlebenden, also deutlich weniger als nach MSÄ- und UV-Behandlung.

Säure-induzierte Mutanten wurden nicht gefunden. Säure wird nur selten als mutagenes Agens benutzt. Sie soll durch Depurinierung der DNS Mutationen hervorrufen ${ }^{21}$. Kaplan und Mitarbb. ${ }^{6}$ erhielten durch Einwirkung von Essigsäure vom $p_{\mathrm{H}}$ 4,6 auf freie Phagen etwa halb soviel Mutanten

19 E. E. Froese-Gertzen, C. F. Konzak, R. Foster u. R. A. Nilax, Nature [London] 198, 447 [1963].

20 E. E. Froese-Gertzen, C. F. Konzak, R. A. Nilan u. R. E. Heiner, Radiat. Botany 4, 61 [1964]. unter den Überlebenden wie nach MSÄ-Einwirkung. Da Hefen von Natur aus ein saures Milieu bevorzugen, hielten wir es für nötig, den $p_{\mathrm{H}}$ Wert drastisch zu senken, damit überhaupt ein Effekt erwartet werden konnte. Trotzdem traten keine Mutanten auf. Da nur 890 Kolonien von Säure-behandelten Zellen geprüft wurden, ist es nicht ausgeschlossen, daß Säure bei Hefen schwach mutagen wirkt. Es kann aber jedenfalls gesagt werden, daß die Säurewirkung in den MSÄ-Versuchen mit verschiedenen $p_{\mathrm{H}}$-Werten bei der Abtötung der Zellen weitgehend und bei der Auslösung von Mutationen vollkommen zu vernachlässigen ist.

Bei Durchsicht der Tab. 2 fällt auf, daß einige Mutantentypen (Methionin) oft auftreten, andere dagegen selten oder gar nicht ( $p$-Aminobenzoesäure). Aus theoretischen Gründen ist anzunehmen, daß diese Verteilung nicht die Verteilung der Mutationsereignisse widerspiegelt. Vielmehr werden wohl alle Mutantentypen zwar induziert, aber nicht immer auch gefunden. Einige Stoffe können z. B. nicht von der Zelle aufgenommen bzw. verwertet werden (Thymin ${ }^{22}$ ), eine entsprechende Mutante würde also nicht auf dem Komplettmedium wachsen und daher nicht gefunden. Andere Stoffe, z. B. Vitamine, könnten in der Zelle in so hoher Konzentration vorliegen, daß die entsprechende Mutante beim Strichtest auf dem Minimalagar noch ein Restwachstum zeigt und daher oft nicht entdeckt wird. Schließlich kann ein Stoffwechselprodukt auf verschiedenerlei Weise gebildet werden: Phenylbrenztraubensäure, die Vorstufe des Phenylalanins, entsteht aus Prephensäure normalerweise enzymatisch, sie kann im sauren $p_{\mathrm{H}}$ des Hefeminimalmediums aber auch rein chemisch durch Umlagerung aus Prephensäure gebildet werden. Eine Phenylalanin-Mutante mit einem genetischen Block vor der Phenylbrenztraubensäure würde also auf Minimalmedium, wenn auch vielleicht verzögert, wachsen können.

Einige von den in Tab. 2 aufgeführten Mutanten können mit mehreren Stoffen wachsen, ohne daß stoffliche oder funktionelle Zusammenhänge zwischen diesen Stoffen erkennbar wären, z. B. wenn entweder Inosit, Leucin oder Valin, Inosit oder Thiamin, Nicotinsäure oder Tyrosin einer Mutante zum Wachstum dienen können. Ähnliche Fälle wur-

21 E. Bautz-Freese, Proc. nat. Acad. Sci. USA 47, 540 [1961].

22 D. H. Williamson, Biochem. J. 90, 25 P [1964]. 
den schon früher bei Hefen ${ }^{23}$ und bei Bakterien ${ }^{16}$ gefunden. Durch den Anteil dieser nur mit Vorbehalten einzuordnenden Mutanten vermindert sich die Zahl der genau klassifizierbaren Mutanten. Wir wollen deshalb davon absehen, die "Mutantenspektren“ der einzelnen verwendeten Mutagene zu durchleuchten.

Die Liste der von uns isolierten Mutanten entspricht im großen und ganzen den schon früher bei Hefen und anderen Organismen gefundenen. Unter den 20 Mutanten, die mit einem Gemisch aller verwendeten Nährstoffe $(, 1-6 “)$ wachsen und unter den 7 Mutanten, die bisher nur auf Komplettmedium zum Wachstum zu bringen waren, könnte eventuell noch weiteres interessantes Material für die Erforschung der Hefe-Biochemie verborgen sein.

Auf Grund der biologischen Tests lassen sich einige Aussagen über die Nicotinsäure-Biosynthese der Hefen machen. Bei den Nicotinsäure-Mutanten förderten Chinolinsäure, 3-Hydroxyanthranilsäure und Kynureninsulfat das Wachstum. Bisher unveröffentlichte Versuche haben gezeigt, daß DL-Tryptophan (Benzolring $\mathrm{U} \cdot{ }^{14} \mathrm{C}$ ) von $S$. cerevisiae in Nicotinsäure umgewandelt wird ${ }^{24}$. Man muß danach annehmen, daß bei $S$. cerevisiae Nicotinsäure aus Tryptophan über die erwähnten Zwischenprodukte gebildet wird. Versuchsergebnisse von SuomalaINEN ${ }^{25}$, der durch Zugabe von 3-Hydroxykynurenin, 3-Hydroxyanthranilsäure und Chinolinsäure die Nicotinsäure-Produktion von Bierhefe steigern konnte, werden dadurch bestätigt. Die früher vertretene Auffassung (l. c. ${ }^{13}$, S. 485 f.), nach der Hefen Nicotinsäure nicht über den „Neurospora(Tryptophan-) Weg“, sondern über den „Bakterien-Weg“ bilden, ist nicht länger haltbar.

Es ist besonders hervorzuheben, daß Chinolinsäure bei Hefemutanten in ebenso geringen Konzentrationen wie Nicotinsäure das Wachstum fördert. Chinolinsäure ist früher bei einer NeurosporaMutante als Akkumulat nachgewiesen worden. Sie hatte jedoch in Versuchen mit Ratten und mit Neurospora keine oder nur geringe ${ }^{26}$ wachstumsfördernde

23 Siehe A. UhL, in: Die Hefen, Bd. I, Herausg. F. ReIfF, R. Kautzmann, H. Lüers u. M. Lindemann, Verlag Hans Carl, Nürnberg 1960.

24 F. Lingens u. P. Vollprecht, Hoppe-Seyler's Z. physiol. Chem., im Druck.

25 H. Suomalainen, Pure appl. Chem. 7, 639 [1963].

26 L. M. Henderson, J. biol. Chemistry 181, 677 [1949].

27 C. E. DAlgliesh, Quart. Rev. [chem. Soc., London] 5, 227 [1951].

28 C. Y YNofsky, in: Amino acid metabolism, ed. W. D. Mc Elroy u. B. Glass, The Johns Hopkins Press, Baltimore 1955.
Wirkung ( $1 \%$ der Wirkung von Nicotinsäure). Aus diesen und anderen Gründen (Literatur s. l. c. ${ }^{27}$ ) blieb es völlig offen, ob Chinolinsäure ein Nebenprodukt oder ein Zwischenprodukt in der Nicotinsäure-Biosynthese ist ${ }^{28-30}$. Erst kürzlich veröffentlichten Gholson und Mitarbb. ${ }^{31}$ Experimente mit Rinderleber-Extrakten, nach denen Chinolinsäure als Zwischenprodukt in der Nicotinsäure-Biosynthese angesehen werden kann. Unsere Versuche mit Hefen und die von Suomalainen ${ }^{25}$ sprechen ebenfalls dafür, wenn man nicht annehmen will, daß in Hefen eine unspezifische Decarboxylase Chinolinsäure in Nicotinsäure umwandelt. Eine weitere Sicherung des Befundes könnten u. a. Chinolinsäure-akkumulierende und nur mit Nicotinsäure wachsende Hefemutanten erbringen, die bis jetzt aber noch nicht isoliert wurden.

Unter den isolierten polyauxotrophen Mutanten sind die zwei Mutanten bemerkenswert, die nur bei gleichzeitiger Gabe von Methionin und Lysin bzw. Threonin und Lysin zu wachsen vermögen. Ein solcher Zweifachbedarf wäre bei Bakterien verständlich, da Methionin, Threonin und Lysin bei Bakterien gemeinsame Vorläufer haben ${ }^{32}$. Die Hefen synthetisieren jedoch Lysin nicht über $\alpha, \varepsilon$-Diaminopimelinsäure wie die Bakterien, sondern über $\alpha$-Aminoadipinsäure. Trotzdem scheint ein Zusammenhang im Stoffwechsel dieser drei Aminosäuren auch bei Hefen zu bestehen, denn Lysin kann die Aspartokinase des Methionin-Threonin-Syntheseweges bei Hefen hemmen ${ }^{33}$. Welcher Art die biochemischen Zusammenhänge sind, läßt sich nicht sagen, besonders deshalb nicht, weil der Biosyntheseweg des Lysins bei Hefen noch nicht genau untersucht ist. Es wäre denkbar, daß die drei Aminosäuren auch bei Hefen gemeinsame Vorläufer haben, und daß Lysin erst in den späteren Syntheseschritten abweichend vom Bakterien-Schema gebildet wird.

Wir danken Fräulein U. Schuster für sorgfältige Mitarbeit und dem Bundesministerium für Wissenschaftliche Forschung für die Bereitstellung von Mitteln.

29 J. G. Morris, in: The Bacteria, Vol. III, ed. I. C. Gunsalus u. R. Y. Stanier, Academic Press, New York und London 1962.

30 V. H. Cheldelin u. A. Baich, in: Biogenesis of Natural Compounds, ed. P. Bernfeld, Pergamon Press, Oxford, London, New York, Paris 1963.

31 R. K. Gholson, I. Unda, N. Ogasawara u. L. M. Henderson, J. biol. Chemistry 239, 1208 [1964].

32 E. R. Stadtman, Bacteriol. Rev. 27, 170 [1963].

33 H. De Robichon-Szulmajster u. D. Corrivaux, Biochim. biophysica Acta [Amsterdam] 73, 248 [1963]. 